Winkeln führen. Diese Atome werden bei unserer Apparatur als gestreute Atome gemessen. Bei Messungen der bekannten klassischen Atomradien wurden nur große Ablenkungswinkel berücksichtigt.

Estermann, Foner und Stern ${ }^{6}$, die ebenfalls mit einer Molekularstrahlapparatur sehr hohen Auflösungsvermögens (5 Bogen-Sekunden) arbeiteten, beobachteten im Einklang mit unseren Resultaten für den Stoß Cs-Cs einen Wirkungsradius von 27,3 A. Messungen anderer Autoren mit ähnlichen Anordnungen beschränkten sich auf Stöße von Kaliumatomen an Edelgasatomen. Ihre Messungen bestätigten die von Massey ${ }^{5}$ für das starrelastische Kugelmodell hergeleitete Gesetzmäßigkeit. Für die Diskussion unserer Ergebnisse ist es wesentlich, daß auf die Atome Zn,
$\mathrm{Cd}$ und $\mathrm{Hg}$ das starrelastische Kugelmodell nicht mehr anwendbar ist und auf Grund der nicht abgesättigten Schalen weitreichende Kräfte und infolgedessen große Wirkungsquerschnitte für Ablenkungen unter kleinen Winkeln zu erwarten sind.

Es fällt auf, daß die gemessenen Wirkungsradien von $\mathrm{Zn}$, Cd und $\mathrm{Hg}$ einen Gang entsprechend ihrer Stellung im periodischen System zeigen. Der Wirkungsradius nimmt von $\mathrm{Zn}$ nach $\mathrm{Hg}$ ab.

Genauere Einzelheiten über die durchgeführten Versuche, die Apparatur, das Berechnungsverfahren und eine Diskussion der Ergebnisse erfolgt in Kürze an einer anderen Stelle.

6 J. Estermann, S. N. Foner u. O. Stern, Phys. Rev. 71, 250 [1947].

\section{Der Anteil der freien Ladungsträger in Germanium an der Absorption im kurzwelligen Ultrarot}

\author{
Von J. Jaumann und R. Kessler
}

\section{Physikalisches Institut der Universität Köln}

(Z. Naturforschg. 9a, 476 [1954]; eingeg. am 6. Mai 1954)

Es ist bekannt, daß im durchsichtigen Gebiet jenseits der Absorptionskante die Absorption von Germanium den nach der Drudeschen Theorie für die freien Ladungsträger der Fremdleitung erwarteten Wert - auch mit quantenmechanischer Korrektur nach Fröhlich - um zwei bis fünf Größenordnungen übertrifft, wohingegen der Frequenzgang über $8 \mu$ ungefähr der Erwartung entspricht. Die von Briggs gefundene genäherte Proportionalität der Absorption mit der Trägerzahl der Fremdleitung von Silicium ist bei Germanium nach Becker und Fan zweifelhaft. Wir haben die Absorption im Temperaturgebiet der Eigenleitung bis $300^{\circ} \mathrm{C}$ im Hochvakuum an p und n Proben mit spez. Widerständen $1 / \sigma$ von 42 bis 0,018 $\mathrm{Ohm} \mathrm{cm}$ (für $20^{\circ} \mathrm{C}$ ) gemessen, wobei Oberflächeneinflüsse durch Messung dreier sehr verschiedener Schichtdicken ausgeschlossen wurden. In diesem Intervall kann man die Trägerzahl und die optische Absorption an demselben Kristall um viele Größenordnungen völlig reversibel mit der Temperatur verändern. Da die Beweglichkeit und vermutlich eventuelle andere Absorptionsursachen weniger als eine Größenordnung schwanken, rührt die Absorptionsänderung größtenteils von der Änderung der Trägerzahl her. Dagegen kann man im bisher untersuchten Temperaturbereich der Fremdleitung die Trägerzahl nur durch Züchtung stark verschieden dotierter Kristalle genügend variieren. Sie ist dann viel kleiner und ihr Einfluß auf die Absorption wird deshalb durch individuelle Unterschiede verschiedener Kristalle fast völlig verdeckt. Das Absorptionsspektrum ist bei allen Proben und Temperaturen strukturlos und von der Bandkante bis $2,8 \mu$ von konstanter Stärke, die mit wachsender Temperatur trotz der Beweglichkeitsänderung und sogar nach dem Übergang zu überwiegender Eigenleitung trotz größenordnungsmäßig erhöhter Trägerzahl, zunächst ungeändert verharrt, dann aber doch steil anwächst und für alle Proben in genau dieselbe Asymptote mit der Aktivierungsenergie $0,756 \mathrm{eV}$ übergeht. Sie entspricht dem erwarteten Wert $(0,752 \mathrm{eV})$ der Aktivierungsenergie des Ausdrucks $1 / R^{2} \sigma$ sehr gut, der für jede Probe und Temperatur durch Messung von $\sigma$ und der HallKonstanten $R$ bestimmt wurde. Das Übergangsgebiet läßt sich sauber in einen temperaturunabhängigen und einen der Asymptote folgenden Teil trennen. Letzteren muß man den Trägern der Eigenleitung zuschreiben. Er übersteigt den theoretischen Wert einheitlich für alle Proben um den Faktor 2300. Bei der Rechnung wurde das Verhältnis von Elektronen- zu Löcherbeweglichkeit gleich 1,5 angenommen. Der temperaturunabhängige Anteil schwankt bei den verschiedenen Proben zwischen 0,4 und $7,9 \mathrm{~cm}^{-1}$. Er erfordert die bekannten, ganz uneinheitlichen, viel höheren Korrekturfaktoren bis $5 \cdot 10^{5}$, wenn man ihn den freien Trägern der Fremdleitung zuordnen will. Er läßt vorläufig keine Beziehung zu Trägerzahl oder Dotierung erkennen. Diese Absorption muß unbekannten Verunreinigungen oder Störstellen zugeschrieben werden und stellt ein viel empfindlicheres Kriterium für solche dar, als elektrische Messungen. Darin ist derAnteil der freien Ladungsträger der Fremdleitung im untersuchten Spektralbereich, den man nach dem Vorhergehenden gut schätzen kann, höchstens bei sehr stark dotierten Proben merklich. Im langwelligen Absorptionsspektrum (etwa über $8 \mu$ ) darf man jedoch wieder ein Hervortreten des Trägeranteils proportional zu $\lambda^{2}$ auch im Temperaturbereich der Fremdleitung und damit wieder eine genäherte Beziehung zwischen Trägerzahl und Absorption sowie den theoretischen Frequenzgang erwarten.

\section{BERICHTIGUNGEN}

Zu L. Holleck und D. E ckardt, Komplexbildung und Termaufspaltung im Absorptionsspektrum Seltener Erden, Band 8a, 660 [1953].

S. $662 \mathrm{a}$, b. Die Unterschriften zu den Abb. 3 und 4 sind vertauscht; die Wellenlängenskalen zu den Abb. 3 und 6 sind verschoben.

Zu L.Holleck und D. Eckardt, Termaufspaltung im Absorptionsspektrum von Erbium und Thulium in Komplexen, Band 9 a, 347 [1954].

S. 640 b. Die seitlichen Beschriftungen der Abb. 2 a und $2 \mathrm{~b}$ sind vertauscht. 